\title{
The molecular gas properties in external galaxies
}

\author{
E. Bayet ${ }^{1}$ S. Viti ${ }^{2}$, M. Bureau ${ }^{1}$, R. Aladro ${ }^{2}$, D. Williams ${ }^{2}$, T. Davis ${ }^{3}$, \\ S. Martin ${ }^{4}$, L. Young ${ }^{5}$, and A. Crocker ${ }^{6}$ \\ ${ }^{1}$ Sub-Dept. of Astrophysics, Dept. of Physics at University of Oxford, Denys Wilkinson \\ Building, Keble Road, Oxford OX1 3RH, U.K. \\ email: bayet@physics.ox.ac.uk \\ ${ }^{2}$ Dept. of Physics and Astronomy, University College London, Kathleen Lonsdale Building, \\ Gower Street, London WC1 6BT, U.K. \\ email: sv@star.ucl.ac.uk \\ ${ }^{3}$ European Southern Observatory, Karl-Schwarzschild-Str. 2, 85748 Garching bei Muenchen, \\ Germany \\ email: tdavis@eso.org \\ ${ }^{4}$ European Southern Observatory, Avda. Alonso de Cordoba 3107, Vitacura, Casilla 19001, \\ Santiago 19, ChileSergio Martin \\ email: smartin@eso.org \\ ${ }^{5}$ New Mexico Tech, 801 Leroy Place, Socorro, NM 8780, USA \\ email: lyoung@physics.nmt.edu \\ ${ }^{6}$ Department of Astronomy, University of Massachusetts, Lederle Graduate Research Tower B \\ 619E, 710 North Pleasant Street, Amherst, MA 01003-9305, USA \\ email: crocker@astro.umass.edu
}

\begin{abstract}
We present a summary of the physical and chemical properties we have obtained for the molecular gas found in galaxies, an essential triggering ingredient to any star formation (SF) process. In particular, we focus our studies on the molecular gas phases as traced by $\mathrm{CO}, \mathrm{HCO}^{+}$and $\mathrm{CS}$ line emissions, in a sample of galaxies including starbursts -SBs-, irregulars galaxies, low-metallicity sources, normal spirals, mergers, Early Type Galaxies (ETGs), galaxies with an Active Galactic Nucleus (AGN), with Cosmic Ray Dominated Regions (CRDRs) and with Photo-Dominated Regions (PDRs). Our studies mix theoretical developments (i.e. computational models) with the acquisition of large observational datatsets (at both low and high spatial resolution) that we then compare in order to better determine the conditions under which some SF activity is starting and/or is maintained.
\end{abstract}

Keywords. astrochemistry — ISM: molecules — submillimeter — stars: formation - ISM: abundances - galaxies: ISM

\section{Introduction}

Interstellar matter in the Universe exists under a plethora of physical and chemical conditions, from tenuous $\left(10^{-5}\right.$ electrons $\left./ \mathrm{cm}^{3}\right)$ hot $(10,000 \mathrm{~K})$ plasma gas to very dense $\left(10^{7} \mathrm{H}\right.$ nuclei $\left./ \mathrm{cm}^{3}\right)$ and cold $(10 \mathrm{~K})$ gas. But not all those gas phases are involved in the star formation processes. To determine whether star formation as we know it in our Galaxy the Milky Way is truly universal, it is essential to study in detail the extragalactic molecular gas phase which is the closest to the stars in formation i.e. the molecular gas. We aim to determine as precisely as possible its properties. To do so, we need to identify first, for a large range of physical and chemical conditions, what are the best tracers (i.e. chemical species line emissions) of the molecular gas phase (see Section 2), then go 
hunting those tracers (see Section 3) to be able to finally derive properties and draw some conclusions (see Section 4).

\section{Modelling work}

The molecular gas is composed of many chemical species, and some can only exist if certain environmental conditions are reached. The emissions of those chemical species, if detected, act thus as powerful diagnostics of the temperature, density, FUV radiation field intensity, etc. The first stage of our work was therefore to identify which are those 'key' molecular species (or combination of thereof) and amongst them, which were likely to be detecteable from ground-based telescopes, in a wide range of conditions. We have thus studied the chemistry of low- and high-density PDRs associated to Giant Molecular Clouds (GMCs) (see Bayet et al. 2009a), of the very dense molecular gas associated with cold and hot cores (see Bayet et al. 2008a) and with the CRDRs (see Bayet et al. 2011b) in normal or enhanced FUV, X-ray and CR radiation fields, mimicking thus normal, SB, AGN and Supernova-dominated environments. We also investigated the chemistry of PDRs, cores and CRDRs when the metallicity was decreased (see references above) or increased (Bayet et al. 2012a). Our results were mostly expressed in fractional abundances but whenever possible we also provided velocity-integrated line intensities (in $\mathrm{K} \mathrm{km} \mathrm{s}^{-1}$ ) and line profiles (see Bayet et al. 2011a).

Our conclusions are numerous but to summarize, it appears that one of the best chemical tracers of the PDR gas especially in SBs are the $\mathrm{HCO}^{+}$line emissions, that the CS line emissions are tracing better the gas found in cores than the HCN line emissions and that we can disentangle CR-induced heating from mechanical or FUV-induced heating by a combination of $\mathrm{CN}, \mathrm{HCO}^{+}$and $\mathrm{C}_{2} \mathrm{H}$ observations. In addition to those new 'key' chemical species are the $\mathrm{CO}$ line emissions, the $\mathrm{CO}$ molecule being the most commonly accepted best chemical tracer of the available gas mass reservoir in galaxies.

\section{Observing work}

In Table 1, we present a summary of the current datasets that we have accumulated since 2002 for the gas reservoir as traced by CO line emissions, for the PDR gas as traced by $\mathrm{HCO}^{+}$line emissions and for the very dense gas as traced by CS line emissions, in both ETGs and late-type galaxies. All the references mentioned in Table 1 also present therein a list of previously published data found in the literature. We refer the reader to those references to get a complete picture of the current status of observational $\mathrm{CO}, \mathrm{HCO}^{+}$ and CS datasets in external galaxies. Papers shown in Table 1 also present isotopologue detections like (e.g.) ${ }^{13} \mathrm{CO}, \mathrm{C}^{34} \mathrm{~S}$ and $\mathrm{H}^{13} \mathrm{CO}^{+}$, ideal species for determining opacity.

\section{Results and Conclusions}

We have derived many line ratios in many galaxies, and from them determine various physical and chemical properties. Amongst them, the most relevant to our study are the gas chemical composition via the determination of fractional abundance ratios of more than 200 species, the gas kinetic temperature, the gas opacity, the $\mathrm{H}_{2}$ volume gas density, the gas column density, and the incident FUV, X-ray and CR fluxes, all three of which can impinge the molecular gas phase with different influence on the chemical composition. One notes that we had a more reliable determination of those properties for late-type galaxies than for ETGs due to more complete datasets (i.e. more numerous molecular transitions and many more isotopologues). 
Table 1. Summary of the observational datasets that we have accumulated for $\mathrm{CO}, \mathrm{HCO}^{+}$ and CS in 18 ETGs and about 10 nearby late-type galaxies. We remind the reader that in the references presented here, we include detailed lists of previously published datasets. Here, we include for late-type galaxies transitions up to $J_{\text {upper }}=7$ while for ETGs, we include transitions up to $\mathrm{J}_{\text {upper }}=3$.

\begin{tabular}{|c|c|c|}
\hline & $\operatorname{ETGs}^{a}$ & late-type galaxies ${ }^{b}$ \\
\hline $\mathrm{CO}$ gas & $\begin{array}{l}\text { Krips et al. }(2010) \text {, Crocker et al. }(2012) \text {, } \\
\text { Alatalo et al. }(2012 \text {, re-submitted) } \\
\text { and Bayet et al. }(2012 \mathrm{~b} \text {, re-submitted })\end{array}$ & $\begin{array}{l}\text { Bayet et al. (2004), Bayet et al. (2006) } \\
\text { and Bayet et al. (2009b) }\end{array}$ \\
\hline $\mathrm{HCO}^{+}$gas & $\begin{array}{l}\text { Krips et al. }(2010), \text { Crocker et al. }(2012)^{c} \\
\text { and Alatalo et al. }(2012, \text { re-submitted })^{c}\end{array}$ & Bayet et al. (2012c, in prep.) \\
\hline CS gas & Davis et al. (2012, in prep.) & $\begin{array}{l}\text { Bayet et al. }(2008 \mathrm{~b}) \text {, Bayet et al. }(2009 \mathrm{c}) \text {, } \\
\text { Aladro et al. }(2011) \text { and } \\
\text { Bayet et al. }(2012 \mathrm{c} \text {, in prep.) }\end{array}$ \\
\hline
\end{tabular}

Notes:

${ }^{a}$ : This sample includes 18 gas-rich ETGs whose detailed list is presented in Bayet et al. (2012b, re-submitted).

${ }^{b}$ : This sample includes SBs (e.g. IC342, M82, NGC253), mergers (i.e. The Antennae Galaxies), Supernovaedominated galaxy (i.e. NGC6946), irregular galaxies (e.g. Henize2-10), low-metallicity source (e.g. IC10), AGNdominated galaxies (e.g. NGC1068), combined SB+AGN-dominated galaxies (e.g. NGC3079), etc.

${ }^{c}$ : Only $\mathrm{J}_{\text {upper }}=1$ emissions have been obtained so far, we expect soon acquire observations of the $\mathrm{HCO}^{+}(3-2)$ line with ALMA in some of those ETGs.

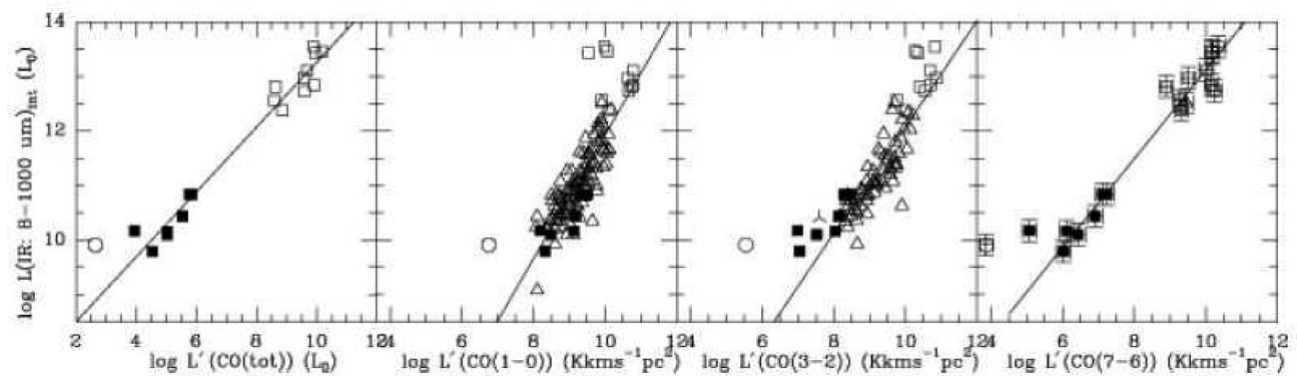

Figure 1. Example of SFR-CO line luminosity relationship (SF laws) we have obtained in Bayet et al. (2009b), i.e. for late-type galaxies (filled squares), high-z galaxies ( $\mathrm{z}=1.439-6.419$, open squares), open triangles representing intermediate-z sources. From left to right, we plot the total integrated IR luminosity $\mathrm{L}_{\mathrm{IR}: 8-1000 \mu \mathrm{m}}$ (in $\left.\mathrm{L}_{\odot}\right)$, the $\mathrm{CO}(1-0), \mathrm{CO}(3-2)$ and $\mathrm{CO}(7-6)$ luminosities (in $\mathrm{Kkms}^{-1} \mathrm{pc}^{2}$ ). The irregular low-metallicity galaxy IC10 is represented by an open circle. The black lines show the linear regression and its slope varies with the CO line studied, showing different relationship between the cooler and more diffuse gas as traced by low-J CO lines and the warmer and denser CO gas as traced by higher-J CO lines. Typical error bars are seen in the extreme right panel. Similar plots are now being obtained for $\mathrm{HCO}^{+}$and $\mathrm{CS}$ in Bayet et al. (2012c, in prep.).

For the CO gas phase, we also provided for the first time the spectral energy line distributions - SLEDs - up to $\mathrm{J}_{\text {upper }}=15$ (see e.g. Bayet et al. $2009 \mathrm{~b}$ and Bayet et al., 2012b, re-submitted.) for late-type galaxies. More recently we also determined for the first time CO SLEDs for ETGs (Bayet et al., 2012b, re-submitted). Interestingly, CO SLEDs for ETGs seem similar to what is seen for the center of the Milky Way. CS SLEDs and $\mathrm{HCO}^{+}$SLEDs for late-type galaxies can be found in Bayet et al. (2012, in prep.) and we show that the position of the SLED turnover whatever the molecule studied seems related to the star formation ( $\mathrm{SF}$ ) rate of the galaxy, providing thus additional useful diagnostic tools for inferring the strength of the SF activity in galaxies. To confirm these results more strongly further studies are needed, especially at very high angular resolution to investigate a potential break-down of this relationship. 

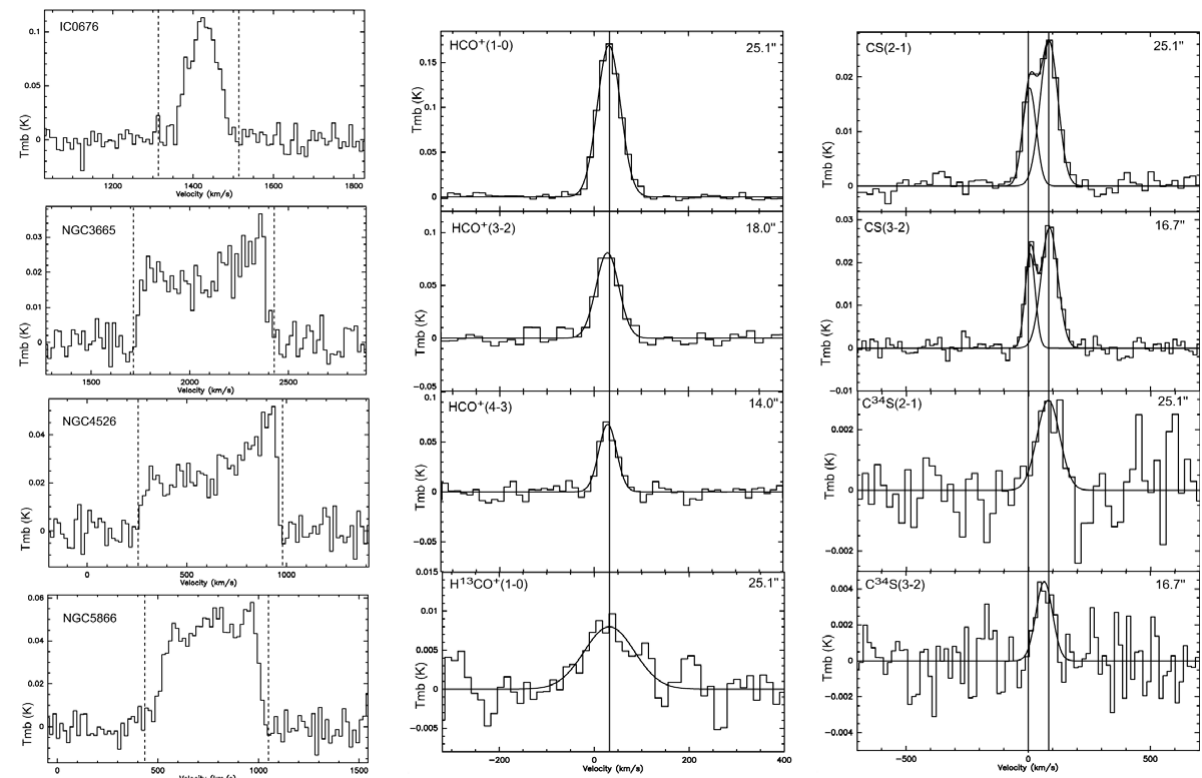

Figure 2. Left: Example of $\mathrm{CO}(3-2)$ line emissions obtained in ETGs (Bayet et al. 2012b, resubmitted), Middle: Example of new multi-line $\mathrm{HCO}^{+}$emissions obtained in the center of IC342 and Right: Example of new multi-line CS line emissions obtained in the center of NGC6946. Middle and right panel are both extracted from Bayet et al. (2012c, in prep.).

As done at low-resolution for the CO gas in late-type galaxies (see Fig.1), we aim at providing now multi-line, multi-molecule, multi-spatial scale star formation laws in many galaxy types, presenting the relationship existing between the total IR luminosity of a galaxy and its low-J and high-J $\mathrm{CO}, \mathrm{HCO}^{+}$and $\mathrm{CS}$ line luminosity. This work will allow us to be able to compare laws for GMCs, PDRs and very dense gas in a large range of galaxy type, bringing therefore significant insight on the contribution of each of those different gas components to the SF activity.

\section{References}

Aladro, R., Martín-Pintado, J., Martín, S., Mauersberger, R., \& Bayet, E. 2011, A\& A, 525, A89 Bayet, E., Gerin, M., Phillips, T. G., \& Contursi, A. 2004, A\&A, 427, 45

Bayet, E., Gerin, M., Phillips, T. G., \& Contursi, A. 2006, A\&A, 460, 467

Bayet, E., Viti, S., Williams, D. A., \& Rawlings, J. M. C. 2008a, ApJ, 676, 978

Bayet, E., Lintott, C., Viti, S., et al. 2008b, ApJL, 685, L35

Bayet, E., Viti, S., Williams, D. A., Rawlings, J. M. C., \& Bell, T. 2009a, ApJ, 696, 1466

Bayet, E., Gerin, M., Phillips, T. G., \& Contursi, A. 2009b, MNRAS, 399, 264

Bayet, E., Aladro, R., Martín, S., Viti, S., \& Martín-Pintado, J. 2009c, ApJ, 707, 126

Bayet, E., Williams, D. A., Hartquist, T. W., \& Viti, S. 2011a, MNRAS, 414, 1583

Bayet, E., Yates, J., \& Viti, S. 2011b, ApJ, 728, 114

Bayet, E., Davis, T. A., Bell, T. A., \& Viti, S. 2012a, MNRAS, 424, 2646

Crocker, A., Krips, M., Bureau, M., et al. 2012, MNRAS, 421, 1298

Krips, M., Crocker, A. F., Bureau, M., Combes, F., \& Young, L. M. 2010, MNRAS, 407, 2261 